\title{
Systematic review of outcome measures in pediatric eosinophilic esophagitis treatment trials
}

Tamar Rubin ${ }^{1}$ (D) Jacqueline Clayton ${ }^{1}$, Denise Adams ${ }^{1,2}$, Rabin Persad ${ }^{1,4}$ and Sunita Vohra ${ }^{1,2,3^{*}}$

\begin{abstract}
Background: Heterogeneity has been noted in the selection and reporting of disease-specific, pediatric outcomes in randomized controlled trials (RCTs). The consequence is invalid results or difficulty comparing results across trials. The primary objective of this systematic review was to assess primary outcome and outcome measure selection and reporting, in pediatric eosinophilic esophagitis (EoE) treatment trials. As secondary objectives, we compared trial disease definition to established concensus guidelines, and the efficacy of current EoE treatments.
\end{abstract}

Methods: We searched MEDLINE, EMBASE, The Cochrane Library, Cochrane Central Register of Controlled Trials (CENTRAL), and CINAHL since 2001. We also searched clinical trial registries (portal.nihr.ac.uk; clinicaltrials.gov; isrctn.com; and anzctr.org.au) and references of included studies. We included RCTs of EoE treatment in patients 0-18 years. Two authors independently assessed articles.

Results: Eleven studies met inclusion criteria. All identified primary outcomes, however, of 9 unique primary outcomes, only 2 were used in more than one study. In total, 25 unique primary and secondary outcome measures were employed for pediatric EoE treatment trials. Measurement properties and rationale for their selection was rarely provided. Uptake of consensus-based diagnostic criteria was $25 \%$ in trials initiated after 2011. Due to the small number and heterogeneity of studies obtained, no meta-analysis of treatment efficacy could be undertaken. This SR was limited to exclusively pediatric RCTs.

Conclusions: The results of this study confirm the need for a standardized set of core outcomes that are universally reported in pediatric EoE trials. Consistent disease definition and standardized outcome reporting will facilitate metaanalyses across similar trials and inform future clinical decision-making.

Systematic review registration number CRD42013003798

Keywords: Eosinophilic esophagitis, Outcome measures, Pediatric, Systematic review, Treatment

\section{Background}

In randomized controlled clinical trials (RCTs,) the primary outcome is "the outcome of greatest importance," [1] and is also the variable that determines calculation of the sample size. Outcome measures, in contrast, are the tools used to measure the primary outcome, and may be scales, questionnaires, scoring systems or other

\footnotetext{
*Correspondence: svohra@ualberta.ca

${ }^{2}$ CARE Program, University of Alberta, Suite \#1702, College Plaza, 8215

112 St NW, Edmonton, AB T6G 2C8, Canada

Full list of author information is available at the end of the article
}

instruments [2, 3]. Although RCTs are universally recognized as the gold standard for determining treatment efficacy, the validity of their results depends on the selection of the most appropriate primary outcomes, valid outcome measurement instruments, and full reporting of the originally stated primary outcomes [4].

A more standardized approach to the selection of outcome measures for disease-specific pediatric RCTs has been proposed as one strategy to help facilitate knowledge synthesis [5]. Standardized outcome selection and reporting, regardless of statistical significance, might also minimize outcome reporting bias [6]. Selective outcome 
reporting is now well accepted as a significant impediment to knowledge translation and meta-analysis [7]. To this end, initiatives such as the Consolidated Standards of Reporting Trials (CONSORT) have been established to help promote transparent and complete reporting $[1,8]$.

In order to facilitate outcome measure selection, the consensus-based standards for the selection of health measurement instruments (COSMIN) group developed an international consensus on the terminology and definitions of measurement properties [9]. They identified three domains of measurement properties: reliability, validity, and responsiveness. Other international initiatives aiming to improve selection and reporting of outcome measures include the COMET initiative (Core Outcome Measures in Effectiveness Trials), which is an initiative to develop a core set of outcome measures for each condition [4].

Methods for appropriate selection of outcome measures in clinical trials have been studied, to some extent, in adults, but very few studies have addressed this problem in children [3]. The validity of outcome measures chosen in pediatric RCTs, as well as the adequacy of their reporting, has been called into question $[2,3,5,10]$. A recent systematic review (SR) of pediatric RCTs found that more than 10 years after CONSORT guidelines were developed, $25 \%$ of pediatric RCTs published in high impact journals still failed to identify a primary outcome [11]. Furthermore, measurement properties of outcome measures were often not reported. Other systematic reviews within specific clinical subspecialities have identified similar problems [12-14].

In order to examine the issues surrounding outcome measure selection and reporting in pediatric RCTs in greater depth, a systematic review within a clinical subspecialty of pediatrics was planned. Eosinophilic esophagitis (EoE) is an immune-mediated inflammatory disease of the esophagus defined by symptoms of esophageal dysfunction and histopathologic findings. This particular condition was strategically chosen as it is a relatively new condition, where many RCTs on the topic would be expected to have been designed well after the development of COSMIN and CONSORT guidelines. Furthermore, heterogenous disease definition in EoE was identified relatively early on, as being an issue in the EoE literature [15]. In 2007, in order to address some of these concerns, the First International Gastrointestinal Eosinophil Research Symposium (FIGERS) published consensus guidelines to help improve treatment and diagnosis of EoE (Table 1) [16]. By 2011, newer updated guidelines, including a revised "conceptual definition" of EoE was developed [17].

The most recent definition states that EoE is a "chronic, immune/antigen-mediated esophageal disease characterized clinically by symptoms related to esophageal dysfunction and histologically by eosinophil-predominant

\section{Table 1 FIGERS criteria for EoE [17]}

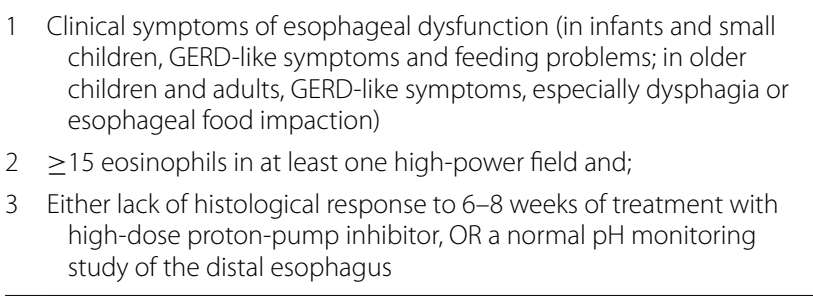

inflammation" [17]. This definition, and its accompanying diagnostic guidelines, emphasizes that both clinical features of esophageal dysfunction, and pathologic features of the disease must be present. The presence of $\geq 15$ Eo/HPF in at least one endoscopic esophageal mucosal biopsy and/or the presence of other microscopic features of eosinophilic inflammation is required for diagnosis. As well, in order to exclude children with PPI-responsive esophageal eosinophilia, an 8-week trial of PPI prior to diagnosis is now also recommended.

The 2011 revisions were a response to certain somewhat arbitrary requirements in the original definition (e.g. histologic finding of 15 or more Eo/HPF), which carry no proven biologic significance or power to discriminate amongst the various esophageal diseases. The requirement to rule out GERD (either via failure of PPI treatment or a normal PH impedance study) had not been rigorously applied to subsequent studies, nor validated. Furthermore, no studies were published since the original consensus report that could allow diagnosis based on a pathognomonic clinical/histologic feature or biomarker.

\section{Objectives}

This SR assessed the heterogeneity of outcome measure selection and reporting in exclusively pediatric EoE treatment RCTs. As secondary objectives, this SR assessed the heterogeneity of definitions of EoE pre- and post-FIGER publication and evidence for current acute treatment modalities for EoE in the pediatric population.

\section{Methods}

This review was registered on PROSPERO prior to the start of the study (CRD42013003798). The search strategy was developed in conjunction with a clinical health research librarian.

\section{Data sources}

We searched MEDLINE, EMBASE, Cochrane Database of Systematic Reviews, Cochrane Central Register of Controlled Trials (CENTRAL), and CINAHL, using all terms relating to EoE. The search was limited to Englishlanguage studies published between January 2001 and December 5, 2014. We also screened the reference lists 
of included studies and searched selected websites for ongoing/registered trials including https://www.portal. nihr.ac.uk/Pages/NRRArchive.aspx; http://www.clinicaltrials.gov/; http://www.isrctn.com/; and http://www. anzctr.org.au/.

\section{Study selection}

Studies were selected if they were: (i) RCTs or controlled trials; (ii) were restricted to pediatric patients (0-18 years) with EoE; (iii) investigated any modality used to treat EoE (e.g. steroids via any route of administration; immune modulating treatment, mast cell inhibitors, monoclonal antibodies; dietary manipulation; esophageal dilatation; novel modalities); and (iv) compared treatment to any control (including, but not limited to, placebo).

Two reviewers (TR, JC) independently screened the abstracts and/or full text of identified articles to determine which ones met criteria. Disagreement was resolved through discussion, including with a senior reviewer (DA, SV) as needed.

\section{Data collection and analysis}

Full texts of all included studies were obtained. Data from included studies were independently extracted by the two reviewers. Disagreement was resolved through discussion, including with a senior reviewer as needed. The following information was extracted: journal name, publication year, design of RCT/CCT, sample size, intervention of interest, number of primary outcomes, outcome measures used, and details of outcome measurement properties. Flexibility in terminology to express "primary outcome" was allowed (e.g. main outcome, primary outcome, end-point etc.). Primary outcomes were examined in detail in order to identify their measurement properties. Information about safety and harms reporting was also extracted.

\section{Assessment of methodological quality of included studies}

The two reviewers independently assessed included studies for risk of bias based on the cochrane risk of bias tool (http://www.cochrane-handbook.org). Where possible, study authors were contacted for additional information. While risk of bias assessment was not necessary to meet the primary objective of our review, it was useful when interpreting data regarding treatment effect.

\section{Results}

A total of 1032 unique references were identified through database searches and another 124 from trial registries. Screening of titles and abstracts excluded 1126 references. Thirty full text articles were obtained and eleven met all inclusion criteria (see Fig. 1). Six were published studies [18-23] and five were registered trial protocols [24-28].

Table 2 provides an overview of the primary outcome measures selected and reported in these studies.

Table 3 provides an overview of the secondary outcome measures selected and reported in these studies.

\section{Outcome measures}

All 11 included studies identified at least one primary outcome with 8 identifying a single primary outcome (Table 2). The number of primary outcomes per study ranged from 1 to 4 .

Nine different primary outcome measures were found and only two were used in more than one study: esophageal eosinophilia (used in 8/11 studies) and safety (used in $2 / 11$ studies). Although many of the studies attempted to assess similar types of primary outcomes (e.g. outcomes that included clinical symptoms), the outcome measures they selected varied: e.g. "physician global assessment score," "EoE clinical symptoms score," "clinical severity score."

Of the 20 different secondary outcomes (Table 3), only 8 occurred in more than one study: Esophageal eosinophilia $(\mathrm{n}=3)$, histologic features $(\mathrm{n}=3)$, proteonomic features $(n=3)$, endoscopic features $(n=2)$, modified endoscopy tool $(\mathrm{n}=2)$, symptom scoring tool $(\mathrm{n}=2)$, immune features $(n=2)$, and adverse events $(n=2)$. Just as in the primary outcome measures, similar endpoints were often being assessed but used different measurement tools. For example, endoscopic features were assessed in at least 4 studies, however outcome measures were different and included "endoscopic features," "modified endoscopy tool," and "severity score for endoscopy and histology." Likewise, symptoms were assessed in multiple studies (at least 6) but used a variety of outcome measures (e.g. "patient symptom report," "clinical response," "symptom scoring tool," "predominant symptom assessment score," and "pediatric EoE symptom severity module").

Combining both primary and secondary outcomes (Table 4), there were 26 unique outcome measures and still only 8 that were used in more than one study: esophageal eosinophilia $(n=9)$, safety $(n=4)$, symptom scoring tool $(\mathrm{n}=3)$, histologic features $(\mathrm{n}=3)$, proteonomic features $(n=3)$, endoscopic features $(n=2)$, endoscopy scoring tool $(n=2)$ and immune features $(n=2)$. Nevertheless, several different outcome measures were often chosen to assess a similar outcome. For example, 9 distinct outcome measures were used to assess clinical symptoms, and at least 4 different outcome measures were used to assess histologic features.

Of the total of 9 unique primary outcomes, 5 (56\%) were scales, scoring systems, instruments, questionnaires 


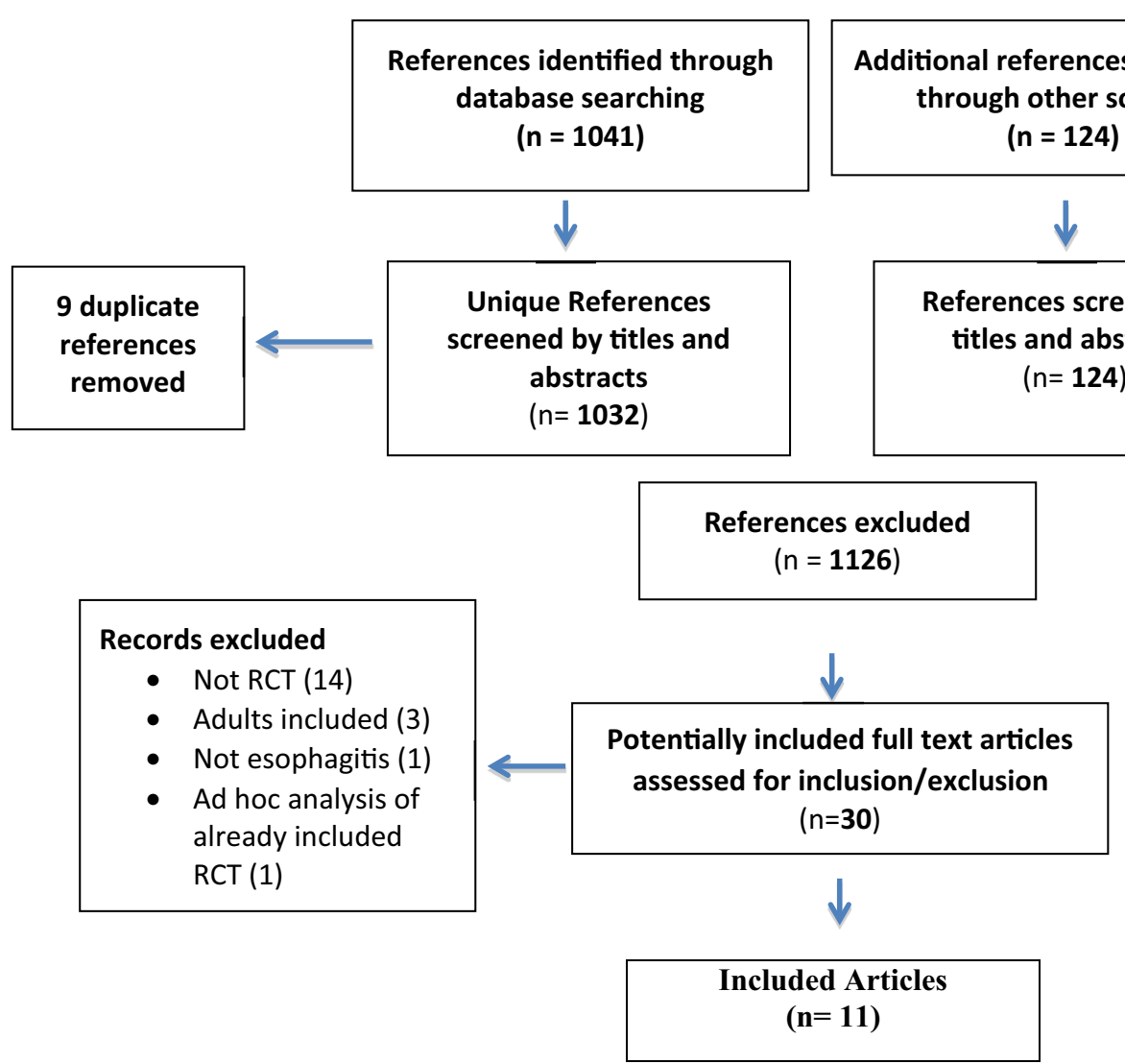

Fig. 1 Flow diagram

or other scoring tools. Of the 25 total primary and secondary outcome measures, 15/25 (60\%) were scales, scoring systems, instruments, questionnaires or other scoring tools. Table 5 summarizes the outcome measurement tools described.

The measurement properties for chosen instruments were only reported in 4/15 cases, and all were unvalidated. Reliability and responsiveness were never reported. Rationale was provided for selection of outcome measures in 6/15 cases, and included "prior use/ reporting of these instruments," "review of available literature", and "ability of the tool to capture a wide range of symptoms specific to pediatric EoE." References were provided for $7 / 15$ of the outcome measurements.

\section{Esophageal eosinophilia}

Although esophageal eosinophilia is a requisite component of the definition of EoE, not all studies measured this as an outcome. Furthermore, outcome measures of esophageal eosinophilia varied significantly in their definitions, methods of measurement, and time period of assessment. For example, some studies defined histologic remission as $\leq 1 \mathrm{Eo} / \mathrm{HPF}$, while other defined remission as $\leq 5 \mathrm{Eo} / \mathrm{HPF}$ or $0-6 \mathrm{Eo} / \mathrm{HPF}$. Other studies assessed percent change in peak Eo counts as evidence of treatment efficacy.

\section{Clinical symptoms}

Clinical symptoms are also a requisite feature of the disease, and were often a component of outcome measurement tools. However, measurement of clinical symptoms in these studies also varied significantly. For example, some of the studies measured combined patient/parent and physician assessments, and others focused on one or the other. Various symptom-scoring tools were also employed.

\section{Disease definition}

Of the 11 trials, 8 were initiated after the publication of the FIGERS criteria. Four out of these eight trials (50\%) met the original FIGERS criteria in their definition of EoE (Table 6). The study by Spergel et al. (and the extension study by Teva Pharmaceuticals) were considered to have met the FIGER criteria for defining EoE, although 


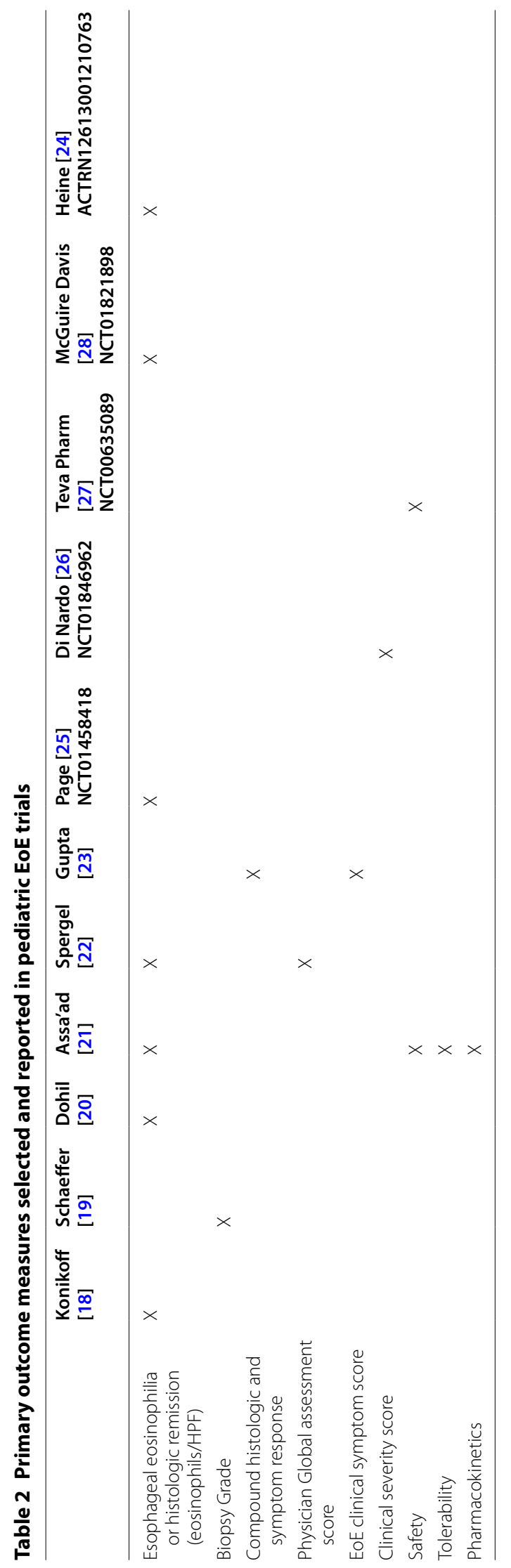




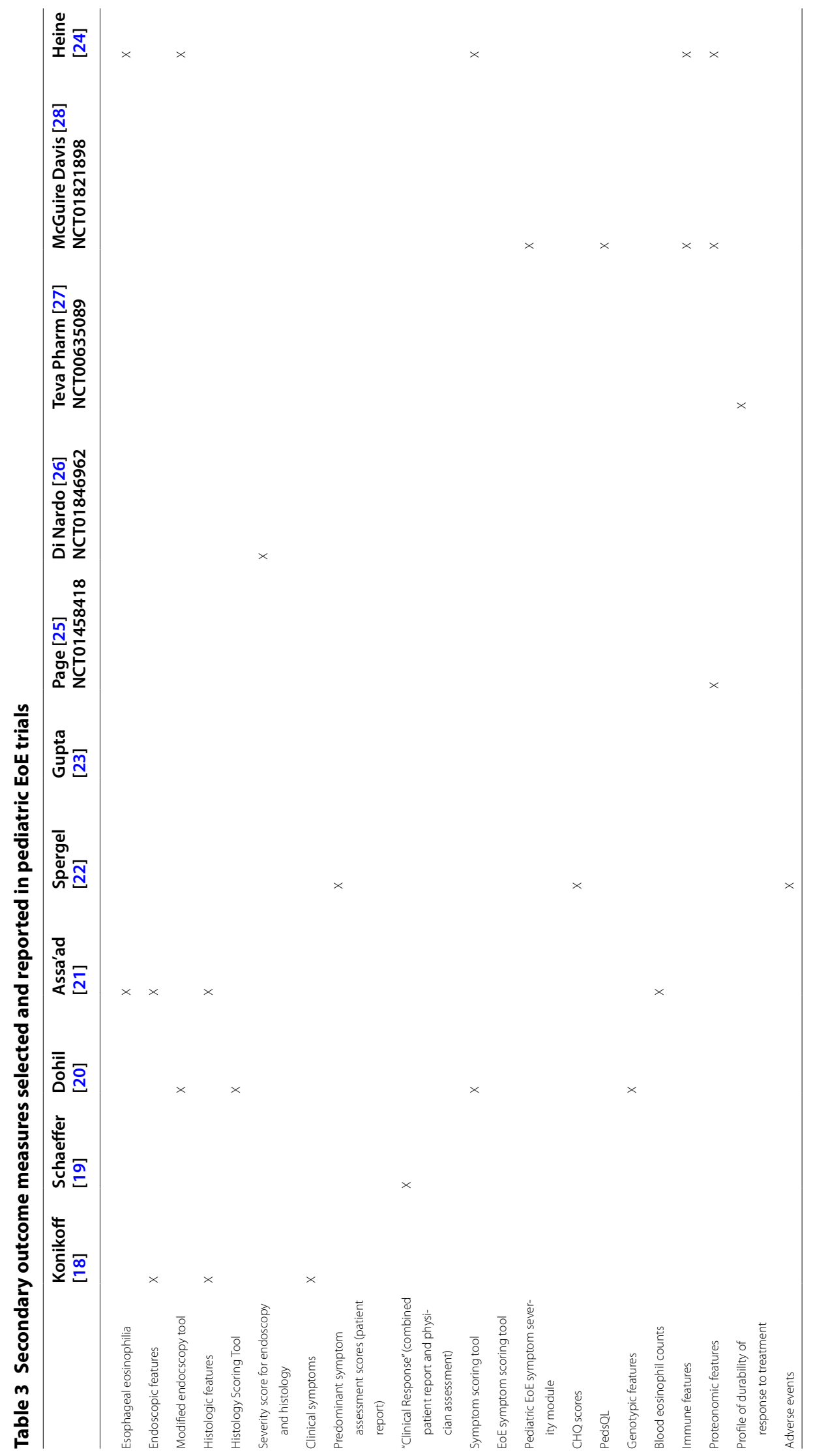




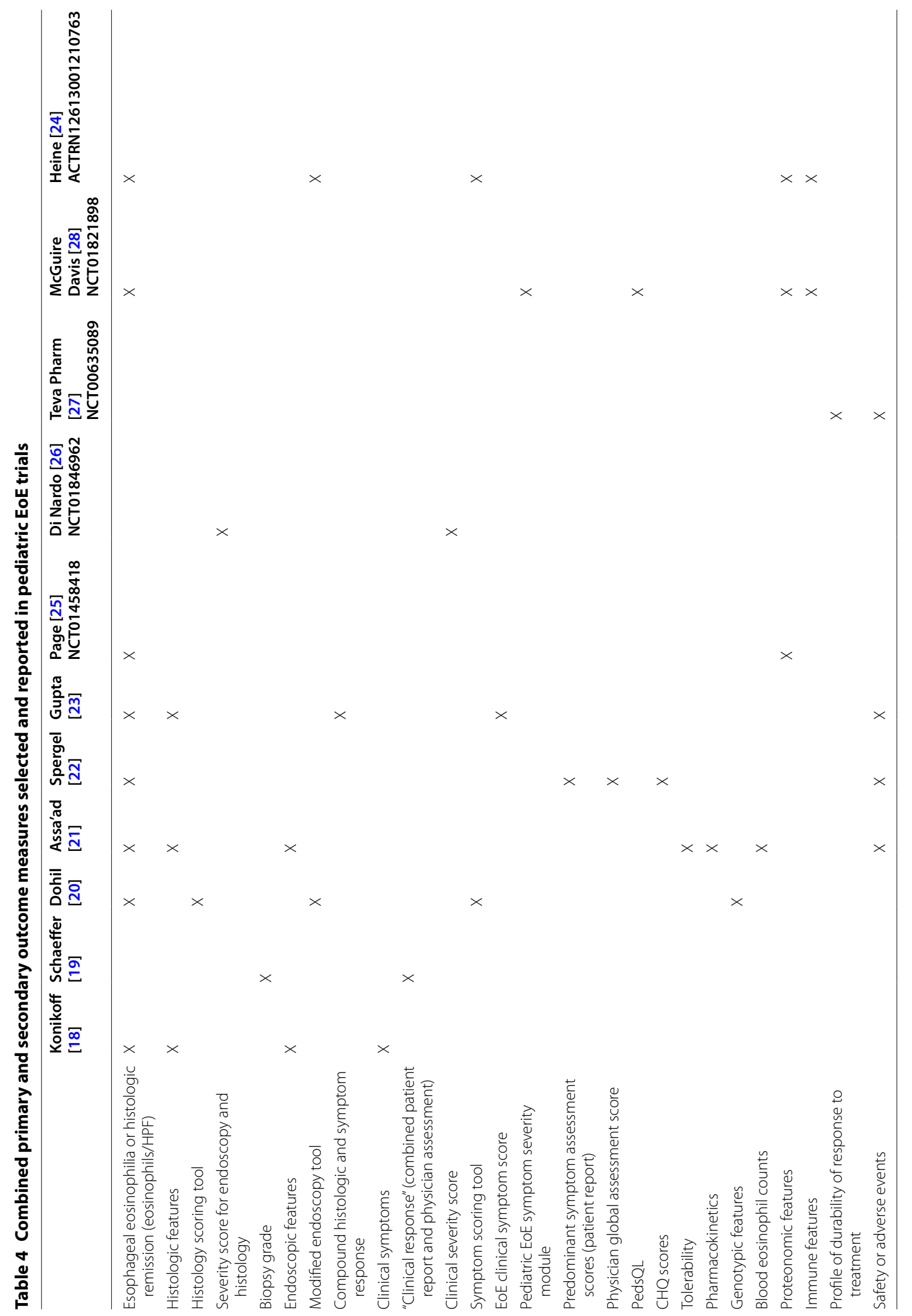




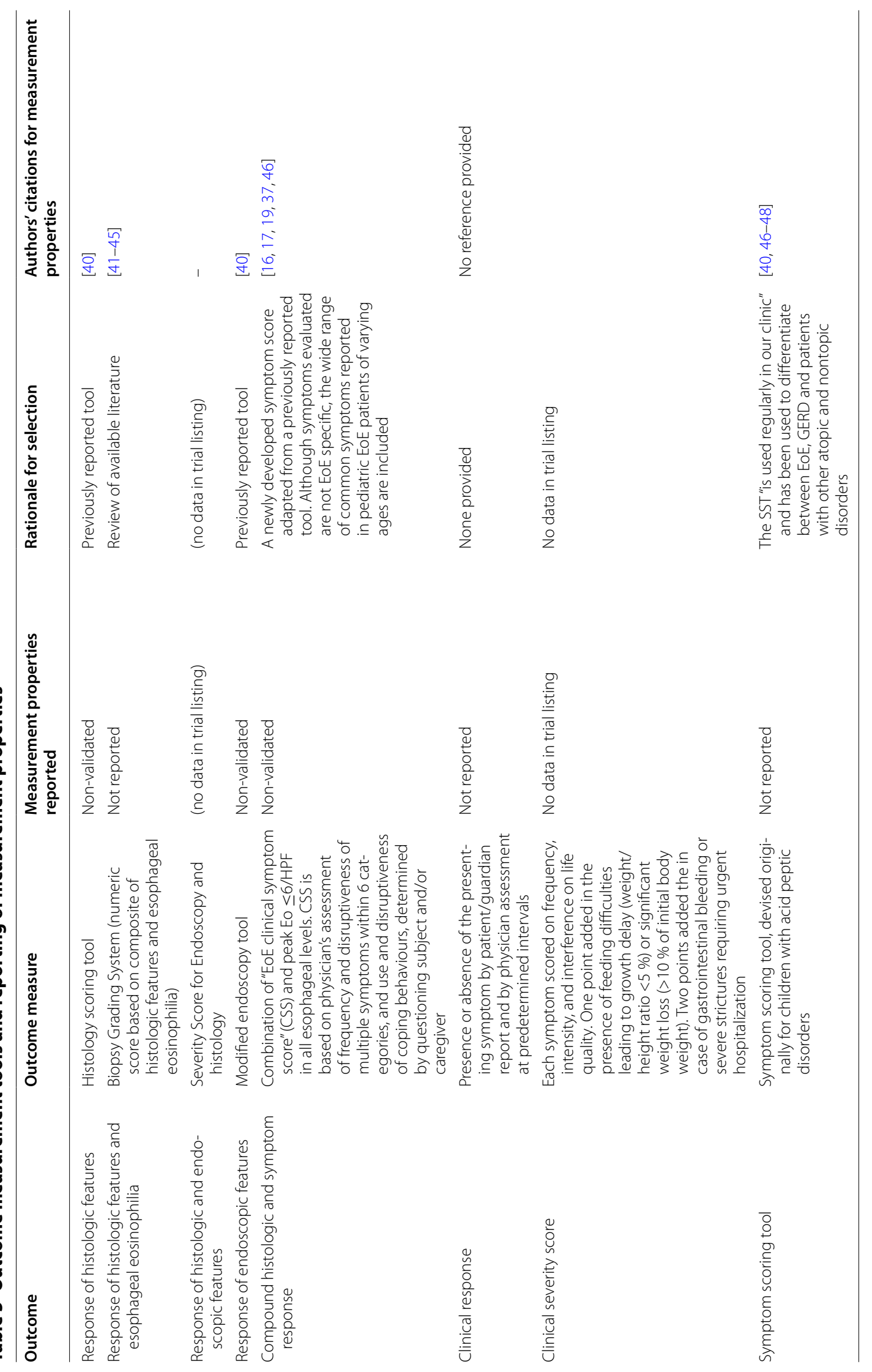




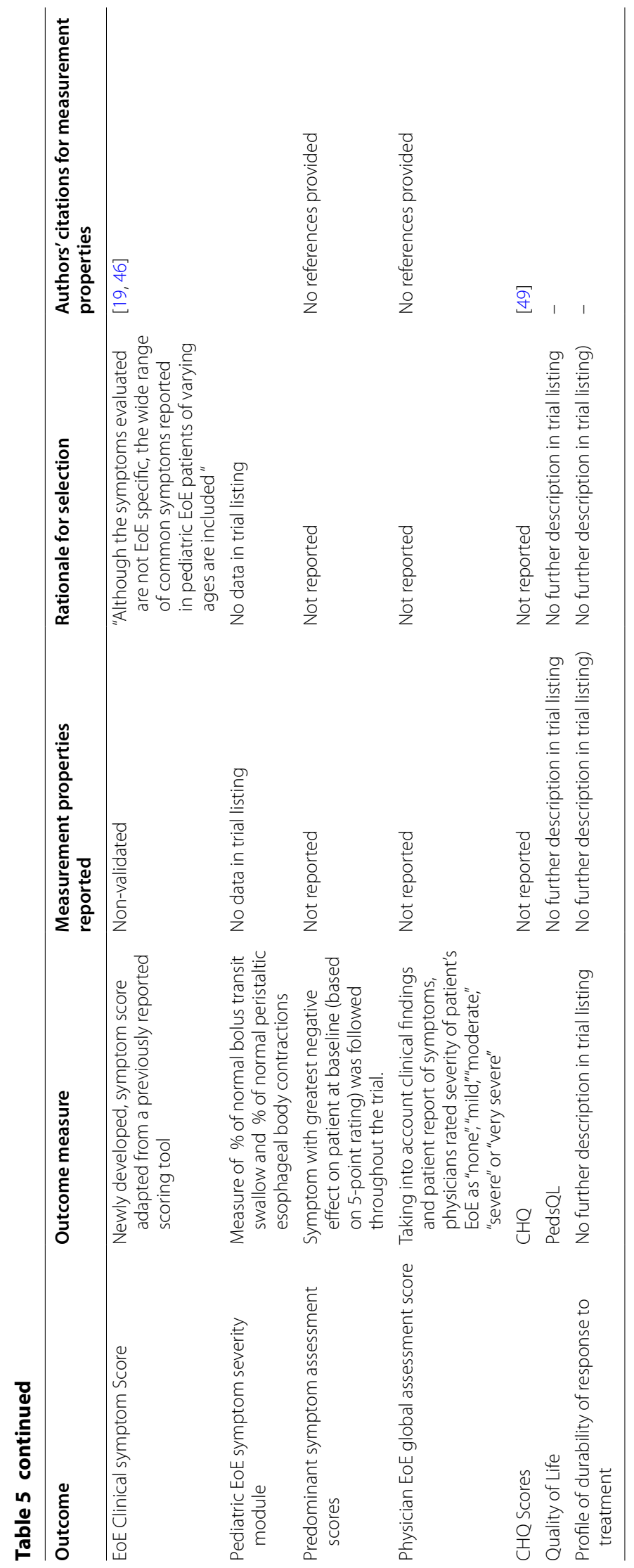




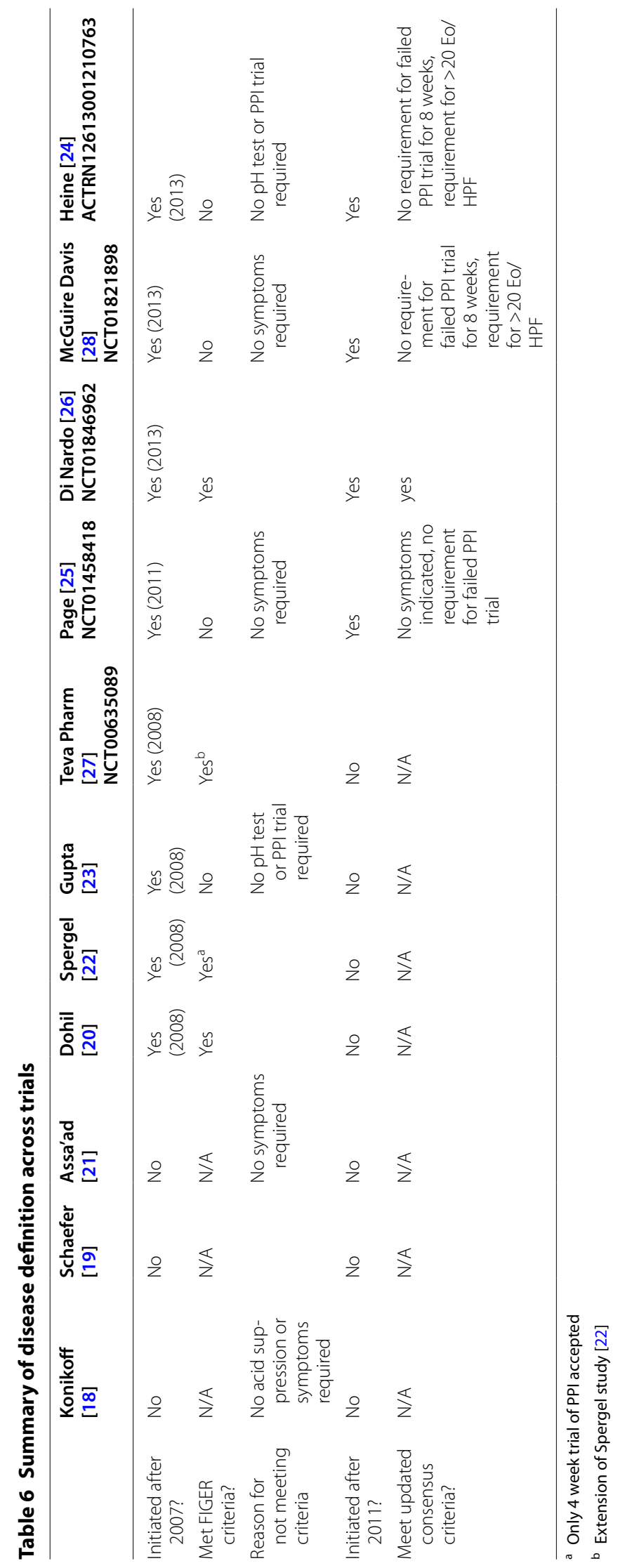


they accepted patients who had failed a 4 week trial of PPI (rather than the 6-8 week required trial outlined in the definition). Of the 4 trials that did not meet FIGER criteria, reasons were: no requirement for symptoms (2), and no requirement for a negative $\mathrm{pH}$ probe or PPI trial (2). Excluding the Spergel trial and its extension study would mean only $2 / 6$ studies met criteria ( $33 \%$ uptake).

Four studies were initiated after publication of the updated EoE consensus guidelines. Only 1 out of 4 ( $25 \%)$ met the new definition for EoE. Three of the studies did not require patients to have failed an 8-week trial of PPI (as suggested in the new guidelines), two required a more stringent definition of esophageal eosinophilia (>20/HPF) than required in the guidelines, and one did not require symptoms.

\section{Treatment efficacy}

Six studies had results available (Table 7). Four studies measured the effect of swallowed steroid [18-20, 23], while 2 studies measured the effect of intravenous antiIL5 therapy [21, 22].

Of the studies measuring swallowed steroid, two assessed swallowed fluticasone, while two assessed swallowed budesonide. The three studies comparing swallowed steroid to placebo found that swallowed steroid was effective in improving histologic features [18, 20,23]. One of those studies found that swallowed oral viscous budesonide was effective in improving symptoms in addition to endoscopic features. Schaeffer compared systemic and topical steroids and found that both were effective in achieving histologic and clinical improvement [19]. Although prednisone seemed to lead to a greater histologic effect, there was no difference observed between systemic and topical steroids in symptom resolution, relapse rate or time to relapse.

Of the two intravenous anti-IL5 trials, both found this agent to reduce intraepithelial esophageal eosinophilia. Spergel et al. found that while intraepithelial esophageal eosinophilia improved with treatment, symptom improvement was observed in all treatment groups, including placebo, and was not associated with changes in the esophageal eosinophilia [22].

Of the 11 identified studies, only 6 were published manuscripts, with relatively low risk of bias overall (see Table 8). Unfortunately intervention and controls were not sufficiently homogenous across studies to allow for comparisons. Meta-analysis in this case is unlikely to provide meaningful data, even across 3 or 4 swallowed steroid trials, or 2 anti-IL 5 studies. A meta-analysis for EoE treatment efficacy was considered; however, given the small number of studies, and heterogeneity in intervention type and outcome measures, this was not feasible.

\section{Discussion}

This systematic review is one in a series of systematic reviews in the PORTal (primary outcomes reporting in trials) initiative, led by Dr. Vohra [11]. In PORTal, RCTs are evaluated to assess how well they report information about primary outcomes and outcome measurement instruments. This systematic review used the PORTal approach to examine these issues in pediatric EoE.

This systematic review identified a handful of exclusively pediatric EoE treatment trials. A number of outcomes were selected and reported in these trials, with certain measures, such as esophageal eosinophilia, clinical symptoms, safety, histologic features, and endoscopic features, re-occurring frequently, but not universally. The rationale for selecting outcome measures, and the measurement properties of the outcome measure tools (when used), were most often not reported. Based on the identified studies, no conclusions regarding treatment efficacy could be made.

\section{Clinical implications}

Prior SRs of EoE treatment suggest a paucity of high quality evidence supporting current treatments for this condition, which, at this time include steroids, immune modulators, dietary modulation, mast cell stabilizers and esophageal dilatation [29,30]. Indeed, much of the pediatric management guidelines for EoE are based on expert opinion and lower quality sources of evidence, such as retrospective observational studies, case reports, and case series [31]. Our current SR of pediatric trials confirms the need for additional treatment RCTs on the topic.

A recent prior SR examined EoE treatment efficacy in RCTs of children and adults up to the year 2010 [29]. While this review demonstrated heterogeneity in outcome measures and disease definition, this was not the focus of the review and these issues were not investigated thoroughly. Furthermore, that review combined adult and pediatric data, neglecting to account for the potentially significant differences in disease presentation, response to treatment, and outcomes, between adult and pediatric populations.

A 2014 SR of dietary treatment for EoE found that dietary interventions are effective in producing histologic remission in patients with EoE [30]. However, Arias et al. combined pediatric and adult data, and included observational studies and case series.

This is not the first time the question has been raised as to whether pediatric and adult EoE are manifestations of a single entity, or two distinct diseases [32, 33]. Notably, pediatric presentations of EoE have been noted to be more heterogeneous, and age-dependent, while in adults 


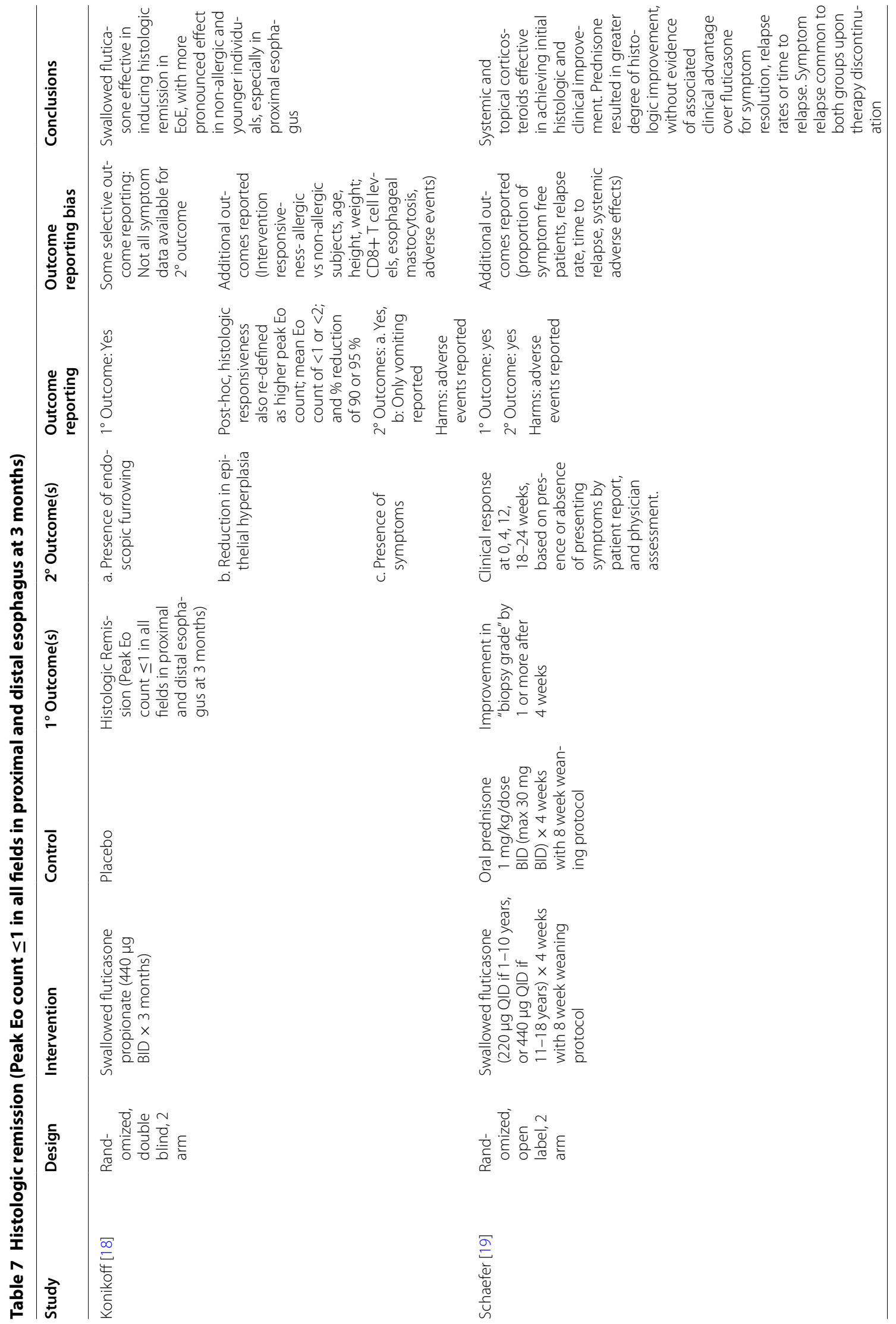




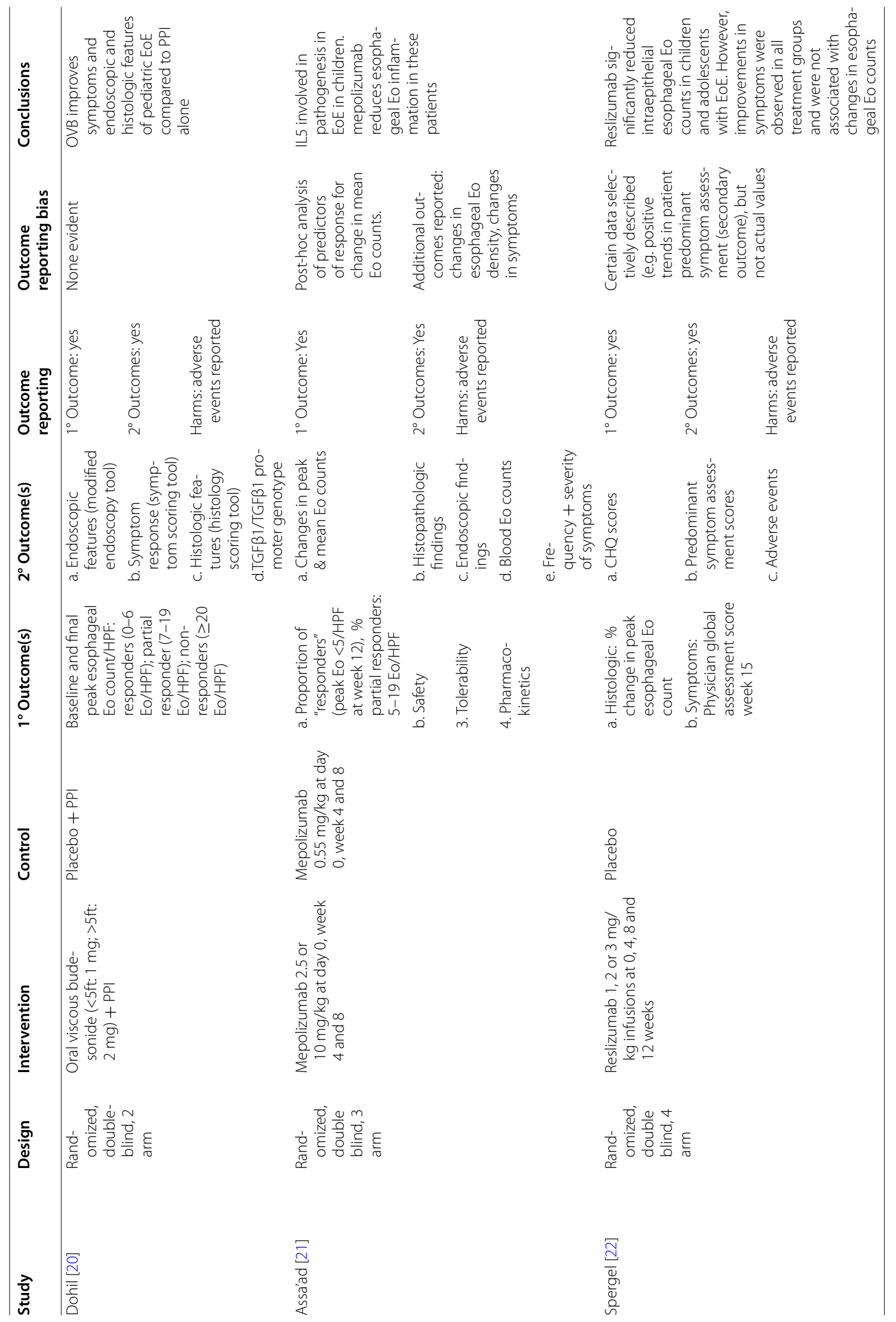




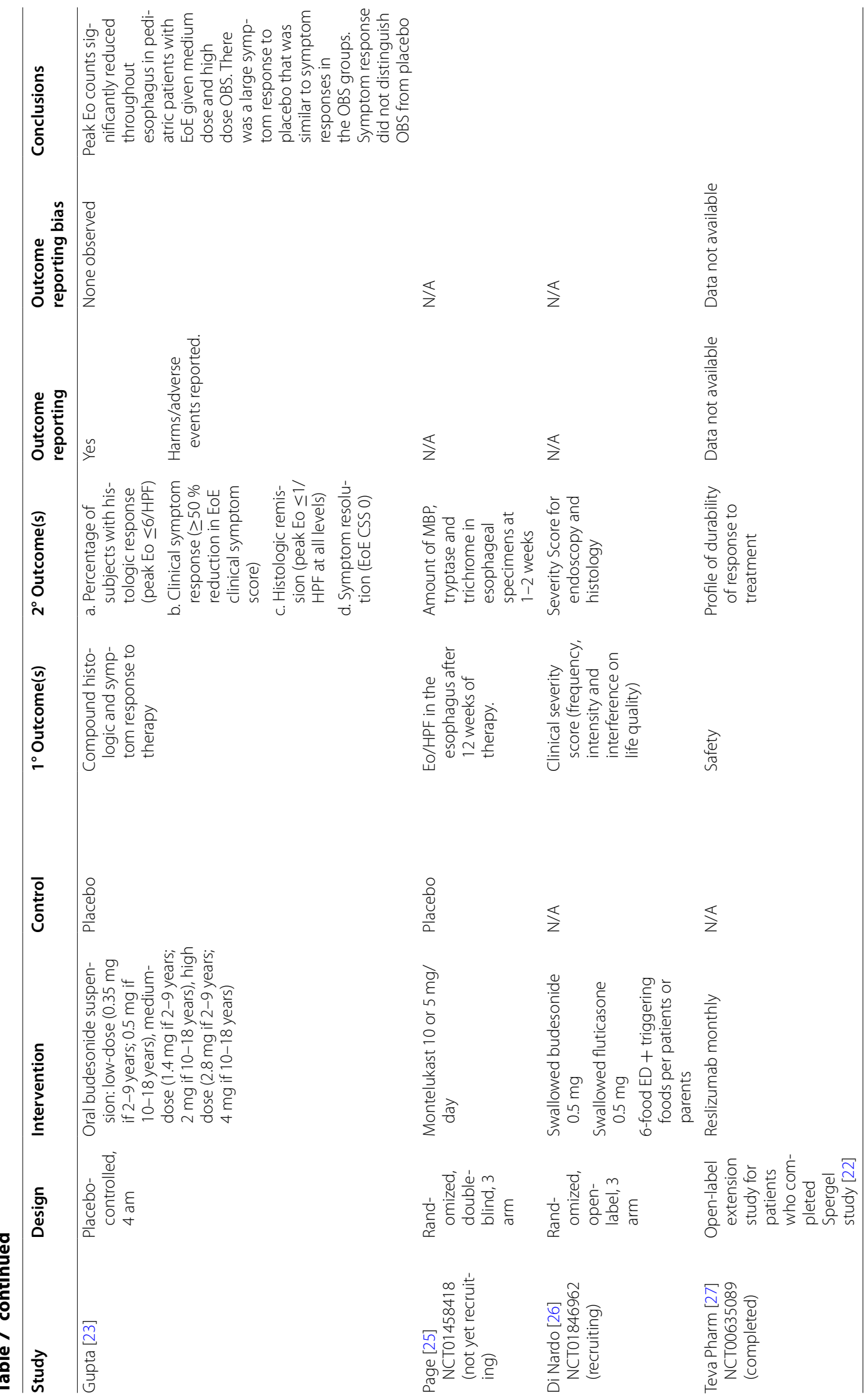




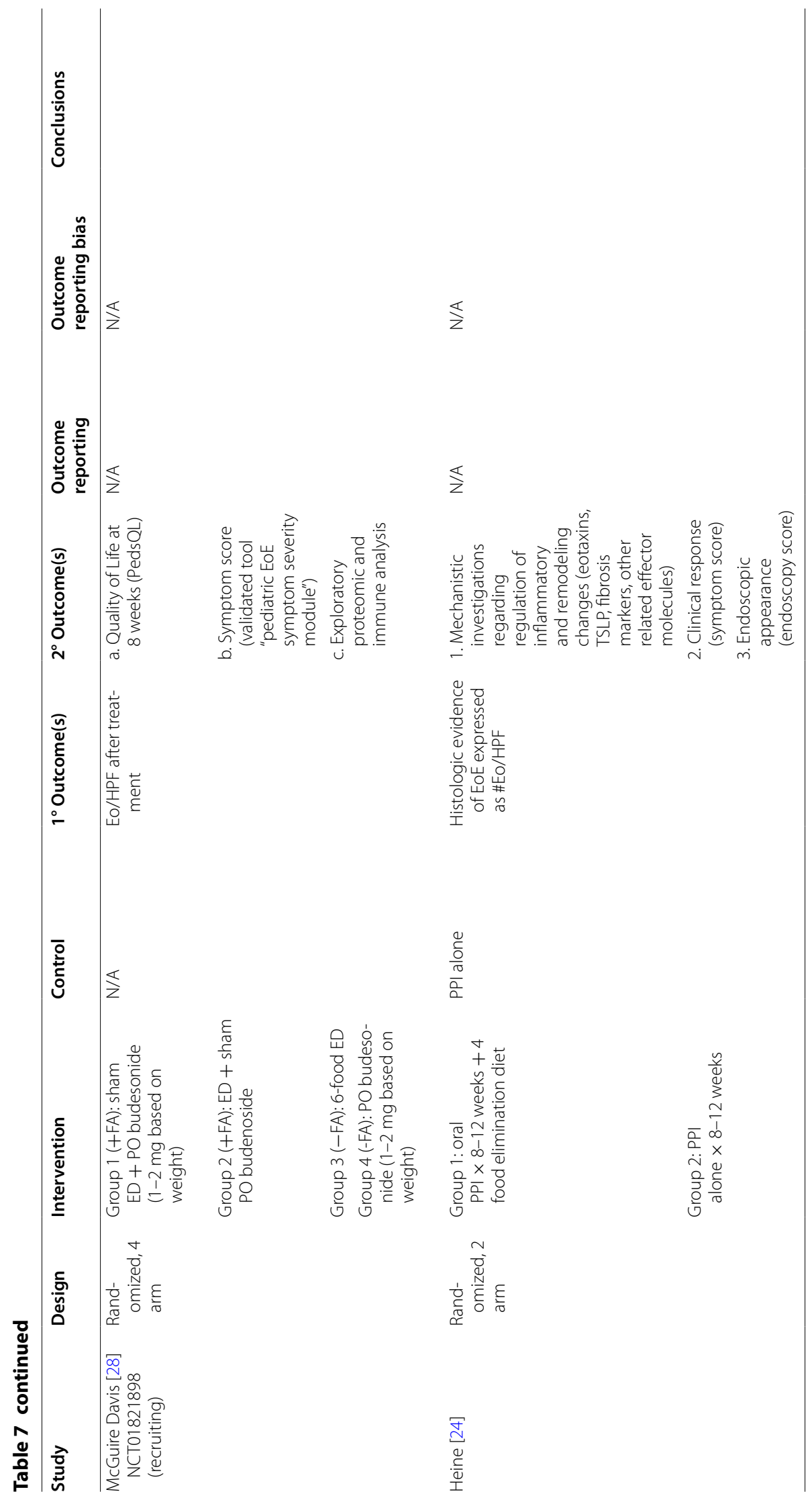


Table 8 Risk of bias assessment in studies

\begin{tabular}{|c|c|c|c|c|c|c|}
\hline & Konikoff [18] & Schaefer [19] & Dohil [20] & Assa'ad [21] & Spergel [22] & Gupta [23] \\
\hline Random sequence generation & + & + & + & $?$ & + & $?$ \\
\hline Allocation concealment & + & + & + & + & + & + \\
\hline Blinding of participants/personnel & + & - & + & + & + & + \\
\hline Blinding of outcome assessment & + & + & + & + & + & $?$ \\
\hline Incomplete outcome data & - & + & - & + & + & + \\
\hline Selective outcome reporting & $?$ & $?$ & + & - & $?$ & + \\
\hline Pharmaceutical sponsorship & + & + & + & - & - & - \\
\hline
\end{tabular}

+ Low risk of bias

-High risk of bias

? Unclear risk of bias

or older adolescents, the clinical presentation tends to be dominated by dysphagia and food impaction [16, 32, 33].

Controversy regarding the most pertinent end points in EoE trials has arisen before [34]. A 2011 editorial by Hirano noted that symptoms and histopathology on endoscopy (generally tissue eosinophilia) have been the most widely used outcomes. The editorial questions, however, whether, indeed, eosinophilia is an adequate or relevant outcome to measure in EoE trials, and urges for the validation of a patient-reported outcome instrument. Our results also find that eosinophilia is the most frequently selected outcome in pediatric trials.

The heterogeneity across outcome measures, and even within individual outcome measures, is not new in this field. Indeed, a 2011 editorial noted that even within commonly used outcomes, such as esophageal eosinophilia, there is variability in the methodology used to quantitate the eosinophils, as well as in the criteria for defining histopathologic change (e.g. reduction in peak eosinophilia versus number of eosinophils per high power field) [34].

Since EoE is being conceptualized as a clinicopathologic disease, experts in the field have emphasized the need for both symptoms and histology to be considered in any therapeutic trial [35]. Some researchers have already suggested that esophageal eosinophilia alone is not a sufficient trial primary end point. Fiorentino et al. suggested using clinical outcome assessment tools such as patient reported outcomes, where possible, with esophageal eosinophilia as a co-primary end point [36].

Significant strides have already been made within EoE research community, in order to address issues of outcome heterogeneity, and the complexity of disease definition for a disease where understanding continues to emerge. Recent American College of Gastroenterology (ACG) guidelines confirm the need for a combination of symptom and pathologic improvement as treatment end points [37]. The development and validation of a novel patient-reported outcome measure of dysphagia in patients with EoE is to be lauded [38].
However, it must be pointed out that a major limitation in the selection of appropriate outcome measures in pediatric EoE trials is our still evolving understanding of the natural history of this disease. Although consensus groups have urged investigators to select and report relevant outcomes, there is still no "gold standard" outcome measure for this disease. Clinical symptoms and histopathologic findings may both turn out to be important for diagnosis and outcomes, as these consensus groups have suggested, but the relative importance of each is not well defined.

Until more information is available regarding the natural history and pathophysiology of the disease, we would suggest that optimal studies in the field should present both clinical and histopathologic data and outcomes. Our systematic review demonstrated heterogeneity in outcome measures and disease definition, and will specifically guide pediatric EoE researchers who aim to design high quality pediatric RCTs in the future.

The uptake of the 2006 FIGERS criteria (50 \%) in pediatric trials in our review is disappointing. Nevertheless, standardization of disease definition across clinical trials is a laudable goal. Another group previously investigated whether 2006 consensus guidelines for EoE diagnosis impacted diagnostic criteria reporting in the literature [39]. They found a significant increase in this reporting in articles published after the release of guidelines compared with those published earlier (31 vs $6 \%, \mathrm{P}<0.001)$.

Of the 4 studies in our SR initiated after the new 2011 guidelines, only 1 adhered to the recommendations. Failure of 8 weeks of a PPI was not a requirement for 3 of the 4 studies. These studies may inadvertently be examining a more heterogeneous population than expected, including patients with PPI-responsive esophageal eosinophilia. Not consistently including or standardizing presenting symptoms of EoE as part of the disease definition makes looking at "clinical symptoms" as an outcome measure challenging. In some cases, patients did not have significant symptoms at baseline, or their symptoms varied 
dramatically at baseline within and between studies. This limits generalizability and comparability of results across studies, since studies included patients with varying degrees of disease severity. Not adequately defining symptoms at the start of the study might also negatively impact on ability to detect change over time.

Similar to the Cochrane review conducted by in 2010, in our SR, no meta-analysis could be conducted due to the limited number of heterogeneous trials identified [29].

\section{Limitations}

The relatively low yield of studies in this systematic review may be related, in part, to the fact that only trials with exclusively pediatric participants were sought. By limiting included studies to those only of children, we optimized the likelihood of age-appropriate outcome measurement instruments being identified. If a study had a mixed adult and pediatric population, it is more likely that outcome measures used may be valid and reliable in one population but not in both (this would potentially disadvantage these studies in our assessment). In addition, there are data to suggest that symptoms, implications, and prognosis of EoE vary between children and adults [32, 33].

\section{Research implications}

Primary and secondary outcome measures selected for the study of EoE varied considerably across treatment trials. No single outcome measure was selected and reported in all trials, which impedes knowledge synthesis. Furthermore, even for outcome measures frequently used (e.g. esophageal eosinophilia, clinical symptoms), standardized methods regarding how and when to assess them were lacking.

In the case of a relatively new and unstudied disease like EoE, validated measurement tools are lacking, which might partly explain the lack of validated measurement instruments being used in the present studies. Effort should be focused on validating measurement tools for use in the pediatric EoE population for future studies. Rationale for selection of outcome measures and appropriate references were rarely provided in these studies.

There is growing data showing that attention to standards for reporting in trials, as in the CONSORT initiative, leads to higher quality RCT reporting [8]. EoE is a relatively new condition and will benefit from research to evaluate which therapies are most effective. Future research would benefit from consistent and standardized definitions of disease occurrence and resolution, and from the development of a core outcome set so that investigators can agree on what outcomes to measure, when, and how.

\section{Conclusions}

The results of this systematic review confirm the need for a core set of standardized pediatric outcome measures that are valid and reliable for future EoE trials. A standardized and rigorous approach to outcome measure selection, such as the COSMIN criteria would be appropriate. Adherence to standardized disease definitions will enhance future knowledge synthesis. Identifying and promoting resolution of heterogeneity in the definition of EoE and its resolution, as well as addressing the issue of heterogeneity in EoE RCT outcome measures, is critical to meaningful knowledge synthesis.

\section{Abbreviations \\ EoE: eosinophilic esophagitis; Eo: eosinophil; SR: systematic review; ACG: American College of Gastroenterology. \\ Authors' contributions \\ TR conceptualized and designed the systematic review, drafted the initial manuscript, and approved the final manuscript as submitted. JC acted as a second reviewer for the systematic review, reviewed and revised the manu- script, and approved the final manuscript as submitted. DA critically reviewed the manuscript, and approved the final manuscript as submitted. RP critically reviewed the manuscript and approved the final manuscript as submitted. SV helped to conceptualize this systematic review, critically reviewed the manuscript, and approved the final manuscript as submitted. All authors read and approved the final manuscript.}

\section{Author details \\ ${ }^{1}$ Department of Pediatrics, University of Alberta, 11405-87 Avenue, 3rd Floor, Edmonton Clinic Health Academy, Edmonton, AB T6G 1C9, Canada. ${ }^{2}$ CARE Program, University of Alberta, Suite \#1702, College Plaza, 8215112 St NW, Edmonton, AB T6G 2C8, Canada. ${ }^{3}$ Department of Public Health Sciences, University of Alberta, 3-300 Edmonton Clinic Health Academy, 11405-87 Ave, Edmonton, AB T6G 1C9, Canada. ${ }^{4}$ Department of Pediatric Gastroenterology and Nutrition, University of Alberta, 11405-87 Avenue, 3rd Floor, Edmonton Clinic Health Academy, Edmonton, AB T6G 1C9, Canada.}

\section{Acknowledgements}

We thank Susanne King-Jones for assistance with article searches.

\section{Competing interests}

The authors declare that they have no competing interests.

\section{Funding}

This work was supported by Alberta Innovates-Health Solutions (AIHS) (formerly AHFMR) and the Canadian Institutes of Health Research. Dr. Vohra receives salary support from AlHS as a health scholar.

Received: 11 May 2016 Accepted: 19 July 2016

Published online: 31 August 2016

\section{References}

1. Schulz KF, Altman DG, Moher D, CONSORT Group. CONSORT 2010 statement: updated guidelines for reporting parallel group randomised trials. BMJ. 2010;2010(340):c332

2. Johnston BC, Shamseer L, da Costa BR, Tsuyuki RT, Vohra S. Measurement issues in trials of pediatric acute diarrheal diseases: a systematic review. Pediatrics. 2010;126:e222-31. 
3. Sinha I, Jones L, Smyth RL, Williamson PR. A systematic review of studies that aim to determine which outcomes to measure in clinical trials in children. PLoS Med. 2008;5(4):e96.

4. The COMET Initiative. http://www.comet-initiative.org. Accessed 16 July 2015.

5. Duncan PW, Jorgensen HS, Wade DT. Outcome measures in acute stroke trials: a systematic review and some recommendations to improve practice. Stroke. 2000;31:1429-38.

6. Williamson PR, Gamble C, Altman DG, Hutton JL. Outcome selection bias in meta analysis. Stat Methods Med Res. 2005;14:515-24.

7. Kirkham JJ, Dwan KM, Altman DG, Gamble C, Dodd S, Smyth R, et al. The impact of outcome reporting bias in randomised controlled trials on a cohort of systematic reviews. BMJ. 2010;340:365.

8. Moher D, Jones A, Lepage L. Use of the CONSORT statement and quality of reports of randomized trials: a comparative before-and-after evaluation. JAMA. 2001;285:1992-5.

9. Mokkink LB, Terwee CB, Patrick DL, Alonso J, Stratford PW, Knol DL, et al. The COSMIN study reached international consensus on taxonomy, terminology, and definitions of measurement properties for health-related patient reported outcomes. J Clin Epidemiol. 2010;63:737-45.

10. Clarke M. Standardising outcomes for clinical trials and systematic reviews. Trials. 2007:8:39.

11. Bhaloo Z. Measurement properties of primary outcome measures in pediatric RCTs: inadequate reporting, insufficient validation, or both? M.Sc. Thesis, University of Alberta Library, 2013.

12. Sinha IP, Williamson PR, Smyth RL. Outcomes in clinical trials of inhaled corticosteroids for children with asthma are narrowly focused on short term disease activity. PLoS One. 2009;4(7):362-76.

13. Reid GT, Walter FM, Brisbane JM, Emery JD. Family history questionnaires designed for clinical use: a systematic review. Publ Health Genom. 2009:12:73-83.

14. Zhang B, Schmidt B. Do we measure the right end points? A systematic review of primary outcomes in recent neonatal randomized clinical trials. J Pediatr. 2001;138(1):76-80

15. Dellon ES, Aderoju A, Woosley JT, Sandler RS, Shaheen NJ. Variability in diagnostic criteria for eosinophilic esophagitis: a systematic review. Am J Gastroenterol. 2007;102(10):2300-13.

16. Furuta GT, Liacouras CA, Collins MH, Gupta SK, Justinich C, Putnam PE, et al. Eosinophilic esophagitis in children and adults: a systematic review and consensus recommendations for diagnosis and treatment. Gastroenterology. 2007;133:1342-63.

17. Liacouras CA, Furuta GT, Hirano I, Atkins D, Attwood SE, Bonis PA, et al. Eosinophilic esophagitis: updated consensus recommendations for children and adults. J Allerg Clin Immunol. 2011:128:3-20.

18. Konikoff MR, Noel RJ, Blanchard C, Kirby C, Jameson SC, Buckmeier BK, et al. A randomized, double-blind, placebo controlled trial of fluticasone propionate for pediatric eosinophilic esophagitis. Gastroenterology. 2006;131(5):1381-91.

19. Schaefer ET, Fitzgerald JF, Molleston JP, Croffie JM, Pfefferkorn MD, Corkins $\mathrm{MR}$, et al. Comparison of oral prednisone and topical fluticasone in the treatment of eosinophilic esophagitis: a randomized trial in children. Clin Gastroenterol Hepatol. 2008;6(2):165-73.

20. Dohil R, Newbury R, Fox L, Bastian J, Aceves S. Oral viscous budesonide is effective in children with eosinophilic esophagitis in a randomized, placebo-controlled trial. Gastroenterology. 2010;139(2):418-29.

21. Assa'ad AH, Gupta SK, Collins MH, Thomson M, Heath AT, Smith DA, et al. An antibody against IL-5 reduces numbers of esophageal intraepithelial eosinophils in children with eosinophilic esophagitis. Gastroenterology. 2011;141(5):1593-604.

22. Spergel JM, Rothenberg ME, Collins MH, Furuta GT, Markowitz JE, Fuchs G 3rd. Reslizumab in children and adolescents with eosinophilic esophagitis: results of a double-blind, randomized, placebo-controlled trial. J Allerg Clin Immunol. 2012;129(2):456-63.

23. Gupta SK, Vitanza JM, Collins MH. Efficacy and safety of oral budesonide suspension in pediatric patients with eosinophilic esophagitis. Clin Gastroenterol Hepatol. 2015;13(1):66-76.

24. Heine. Database: anzctr.org.au/. 2013. https://www.anzctr.org.au/Trial/ Registration/TrialReview.aspx?id=363865Accessed 2 Jan 2015.

25. Page S. Database: Clinicaltrials.gov. 2011. http://www.clinicaltrials.gov/ show/NCT01458418 Accessed 19 Sept 2014
26. Di Nardo G. Database: Clinicaltrials.gov. 2012. http://www.clinicaltrials. gov/show/NCT01846962 Accessed 19 Sept 2014.

27. Teva Pharm. Database: Clinicaltrials.gov. 2013. http://www.clinicaltrials. gov/show/NCT00635089 Accessed 19 Sept 2014.

28. McGuire Davis C. Database: Clinicaltrials.gov. 2013. http://www.clinicaltrials.gov/show/NCT01821898 Accessed 19 Sept 2014.

29. Elliott EJ, Thomas D, Markowitz JE. Non-surgical interventions for eosinophilic esophagitis. Cochrane Database Syst Rev. 2010;3:CD004065.

30. Arias A, González-Cervera J, Tenias JM, Lucendo AJ. Efficacy of dietary interventions for inducing histologic remission in patients with eosinophilic esophagitis: a systematic review and meta-analysis. Gastroenterology. 2014;146:1639-48.

31. Papadopoulou A, Koletzko S, Heuschkel R, Dias JA, Allen KJ, Murch SH, et al. Management guidelines of eosinophilic esophagitis in childhood. J Pediatr Gastroenterol Nutr. 2014;58(1):107-18.

32. Straumann A, Aceves SS, Blanchard C, Collins MH, Furuta G, Hirano I, et al. Pediatric and adult eosinophilic esophagitis: similarities and differences. Allergy. 2012;67:477-90

33. Lucendo A, Sánchez-Cazalilla M. Adult versus pediatric eosinophilic esophagitis: important differences and similarities for the clinician to understand. Expert Rev Clin Immunol. 2012;8(8):733-45.

34. Hurano I. Therapeutic end points in eosinophilic esophagitis: is elimination of esophageal eosinophils enough? Clin Gastroenterol Hepatol. 2012;10(7):750-2.

35. Rothberg ME, Aceves S, Bonis PA, Collins MS, Gonsalves N, Gupta SK. Working with the US food and drug administration: progress and timelines in understanding and treating patients with eosinophilic esophagitis. J Allerg Clin Immunol. 2012;130(3):617-9.

36. Fiorentino R, Liu G, Pariser A, Mulberg AE. Cross-sector sponsorship of research in eosinophilic esophagitis: a collaborative model for rational drug development in a rare disease. J Allerg Clin Immunol. 2012;130:613-6.

37. Dellon ES, Gonsalves N, Hirano I, Furuta GT, Liacouras CA, Katzka DA, American College of Gastroenterology. ACG clinical guideline: evidence based approach to the diagnosis and management of esophageal eosinophilia and eosinophilic esophagitis (EOE). Am J Gastroenterol. 2013:108:679-92.

38. Dellon ES, Irani AM, Hill MR, Hirano I. Development and field testing of a novel patient-reported outcome measure of dysphagia in patients with eosinophilic esophagitis. Aliment Pharm Ther. 2013;38(6):634-42.

39. Sperry $S$, Shaheen N, Delon E. Toward uniformity in the diagnosis of eosinophilic esophagitis (EoE): the effect of guidelines on variability of diagnostic criteria for EoE. Am J Gastroenterol. 2011;106:824-32.

40. Aceves S, Newbury R, Dohil M, Bastian J, Dohil R. A symptom scoring tool for identifying pediatric patients with eosinophilic esophagitis and correlating symptoms with inflammation. Ann Allerg Asthma Immunol. 2009;103:401-6.

41. Kelly KJ, Lazenby AJ, Rowe PC, Yardley JH, Perman JA, Sampson HA. Eosinophilic esophagitis attributed to gastroesophageal reflux: improvement with an amino acid-based formula. Gastroenterology. 1995;109:1503-12.

42. Knuff TE, Benjamin SB, Worsham GF, Hancock JE, Castell DO. Histologic evaluation of chronic gastroesophageal reflux. An evaluation of biopsy methods and diagnostic criteria. Dig Dis Sci. 1984;29:194-201.

43. Hyams JS, Ricci A Jr, Leichtner AM. Clinical and laboratory correlates of esophagitis in young children. J Pediatr Gastroenterol Nutr. 1988;7:52-6.

44. Shub MD, Ulshen MH, Hargrove CB, Siegal GP, Groben PA, Askin FB. Esophagitis: a frequent consequence of gastroesphageal reflux in infancy. J Pediatr. 1985;107:881-4.

45. Goldman H, Antonioli DA. Mucosal biopsy of the esophagus, stomach and proximal duodenum. Hum Pathol. 1982;13:423-48.

46. Aceves SS, Bastian JF, Newbury RO, Dohil R. Oral viscous budesonide: a potential new therapy for eosinophilic esophagitis in children. Am J Gastroenterol. 2007:102:1-9.

47. Dohil R, Newbury RO, Sellers ZM, Deutsch R, Schneider JA. The evaluation and treatment of gastrointestinal disease in children with cystinosis receiving cysteamine. J Pediatr. 2003;143:224-30.

48. Dohil R, Fidler M, Barshop B, Newbury R, Sellers Z, Deutsch R, et al. Esomeprazole therapy for gastric acid hypersecretion in children with cystinosis. Pediatr Nephrol. 2005;20:1786-93.

49. Landgraf JM, Abetz L, Ware JE. The CHQ user's manual. Boston: The Health Institute, New England Medical Center; 1996. 